\title{
Anerobic Spondylodiscitis: A Case Report
}

\author{
Mohammed Kashab ${ }^{\mathrm{a}}$ e , Mohammed Khalifa ${ }^{\mathrm{a}}$, Hatim M. Alshareef ${ }^{\mathrm{b}}$, Amer M. Alshareef $^{\mathrm{c}}$, \\ Ahmed H. Alhasan ${ }^{\text {, }}$
}

\begin{abstract}
A 60-year-old female patient with a history of hypertension, diabetes mellitus, major depression, dyslipidemia, hypothyroidism, osteoarthritis and minor thalassemia on medications presented to the hospital complaining of progressive lower limb weakness over the past 10 days. An epidural injection was done as a pain control measure. She was diagnosed as anerobic spondylodiscitis. This case is unique due to the abrupt progression of the clinical picture from non to paraplegia. It is also unique in the severe destructive picture seen in the CT and MRI images done as diagnostic measures, keeping in mind that these investigations were done less than 3 weeks after the injection.
\end{abstract}

Keywords: Anerobic; Spondylodiscitis; Paraplegia

\section{Introduction}

We report a case of anerobic spondylodiscitis that took place following an epidural injection done as a pain control measure. This case is unique due to the abrupt progression of the clinical picture from non to paraplegia. It is also unique in the severe destructive picture seen in the CT and MRI images done as diagnostic measures, keeping in mind that these investigations were done less than 3 weeks after the injection.

\section{Case Report}

A 60-year-old lady with known conditions of hypertension, di-

Manuscript accepted for publication August 10, 2016

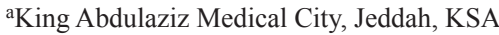

${ }^{b}$ King Fahd Armed Forces Hospital, Jeddah, KSA

'Ibn Sina National College, Jeddah, KSA

dUniversity of Jeddah, Jeddah, KSA

eCorresponding Author: Mohammed Kashab, King Abdulaziz Medical City, Jeddah, KSA. Email: Dr.mohammed.kashab@hotmail.com; Hatim M. Alshareef, King Fahd Armed Forces Hospital, Jeddah, KSA.

Email: dr.hatim.alshareef@gmail.com abetes mellitus, major depression, dyslipidemia, hypothyroidism, osteoarthritis and minor thalassemia on medications presented to the hospital complaining of progressive lower limb weakness over the past 10 days. She gave a history of low back pain as a result of her prolapsed disk which was relieved by an epidural analgesic injection composed of steroid and local anesthetic in King Khalid Hospital, National Guard, Jeddah (KKH, NGHA, Jeddah) 3 weeks before her presenting illness. The injection was at the L4-L5 level. Her symptoms abruptly progressed from tingling sensations in her lower limbs until the patient completely lost motor function in both lower limbs. There was no history of trauma or constitutional symptoms. She had a neurogenic bladder at presentation.

The patient had history of previous similar epidural injections for her back pain. She had past surgical history for hernia repair. Also, she had past allergic history to penicillin and fish.

Upon admission, the patient was afebrile, and her vital signs were stable; generally she was conscious, alert, oriented and looked depressed. She was morbidly obese (BMI 51.6). Chest, cardiovascular and abdomen examinations showed no abnormality. Neurological examination showed lower limb muscle

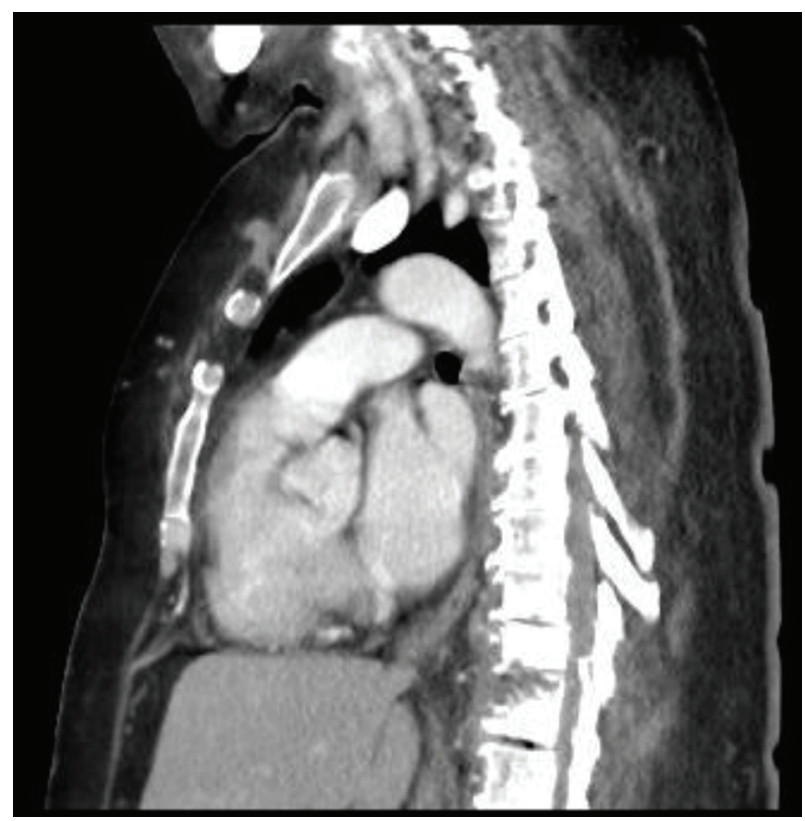

Figure 1. Pathological fracture at the T9-T10 level. 


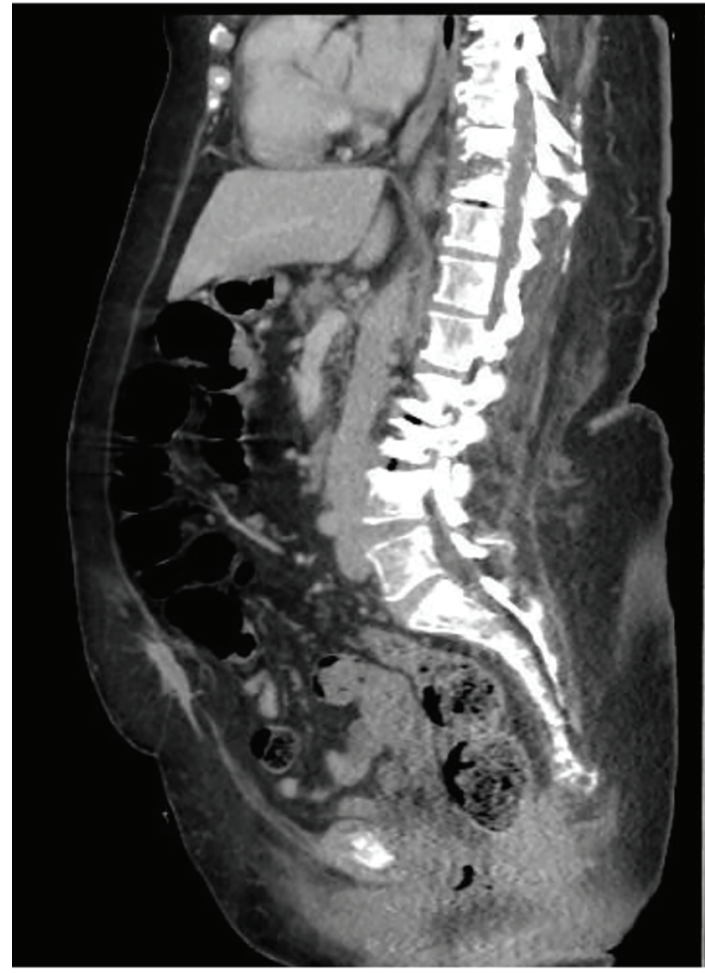

Figure 2. Segmental kyphosis and severe canal stenosis at the T9-T10 level.

power of $0 / 5$, absent sensation and reflexes from the T10 level and downwards. The Babinski sign was negative. There was tenderness in her back at the level of T9-T10. The admission impression was spinal cord compression secondary to epidural hematoma after the epidural analgesic injection she received.

CT was done urgently upon suspicion of post-injection hematoma. It showed no pathology at the level of injection. Rather it showed a pathological fracture at the T9-T10 level with segmental kyphosis and severe canal stenosis as shown in Figures 1 and 2. MRI images/tests indicated spondylodiscitis, cord compression and myelomalacia at T9-T10 and canal stenosis as shown in Figure 3.

WBCs were 14.4 cells $/ \mathrm{mm}^{3}$, CRP was 81.1, and ESR was $37 \mathrm{~mm} / \mathrm{h}$. A CT-guided biopsy did not demonstrate the presence of any organism but the cell count was 40,600 (86\% neutrophils). The patient was put on an antibiotic regime prescribed empirically in the form of vancomycin $1 \mathrm{~g} \mathrm{q} 12 \mathrm{~h}$ and ceftriaxone $2 \mathrm{~g}$ q $24 \mathrm{~h}$. Surgery was done for the patient 4 days after admission as an urgent procedure to decompress T9, T10 with fusion and stabilization using transpedicular screws. It included multiple levels decompression with laminectomy from T6 down to T12 and posterior instrumentation.

Intraoperative findings were segmental kyphosis at the T9-T10 level with epidural fat necrosis. Upon decompressing the same level, a transpedicular biopsy showed serogenous fluid with bone debris and no pus.

There was also reactive new bone formation. There was no evidence of pus. An open biopsy for the T10 lesion was taken. Multiple samples were taken for histopathology including a

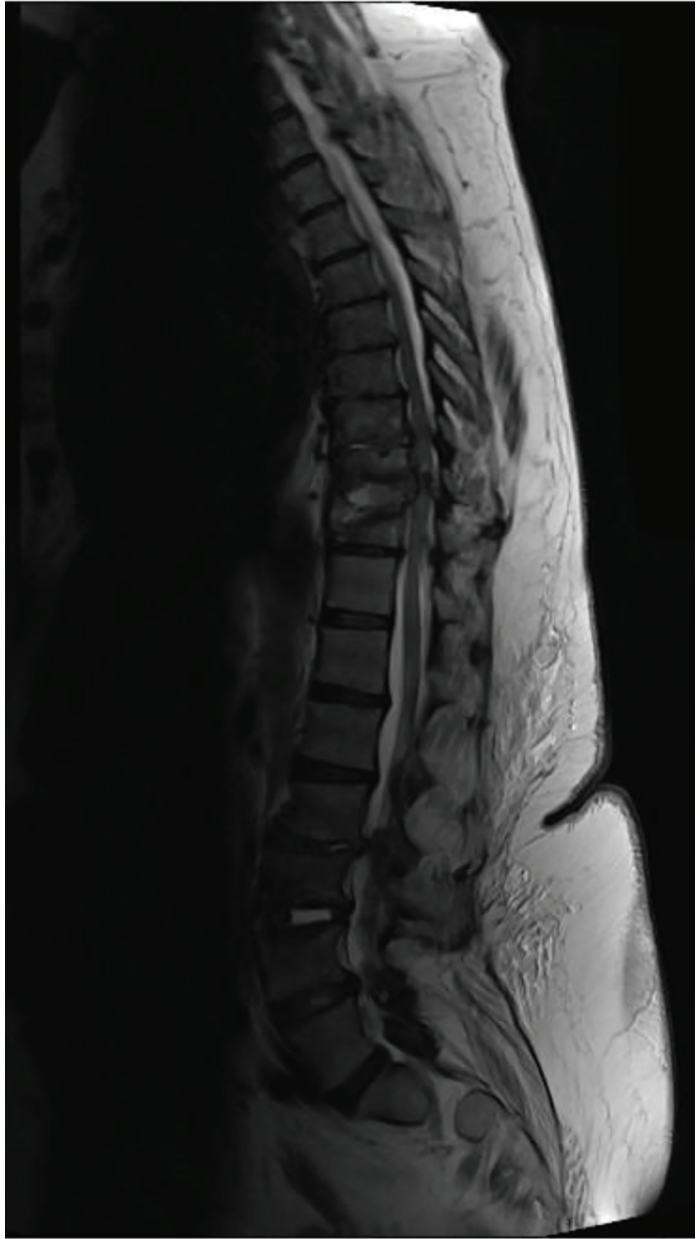

Figure 3. Spondylodiscitis, cord compression and myelomalacia at T9T10 with canal stenosis.

dry sample, a wet sample in saline and formalin, as well as fluid for cytology. Multiple cultures were tested for aerobic and anerobic organisms, as well as TB, brucella and fungal culture.

The results of the microbiology tissue culture indicated that there was positive peptococcus infection of the vertebral bone.

After observing the offending organism and sensitivity study, the antibiotics were changed to ceftriaxone $2 \mathrm{~g}$ IV daily and metronidazole $500 \mathrm{mg} \mathrm{IV} \mathrm{q12h.} \mathrm{The} \mathrm{patient} \mathrm{was} \mathrm{followed}$ up by series of blood works which demonstrated all parameters going back to normal values within a 3-month period. After 4 months period of the patient's presentation, her motor function did not recover. A follow-up MRI was done 4 months after the surgery and it showed complete resolution of the infection and an acceptable fusion of the T9-T10 (Fig. 4).

\section{Discussion}

There were no difficulties in establishing the diagnosis in our reported case. What was unique was the abrupt progression of the clinical presentation. Three scenarios were structured upon 


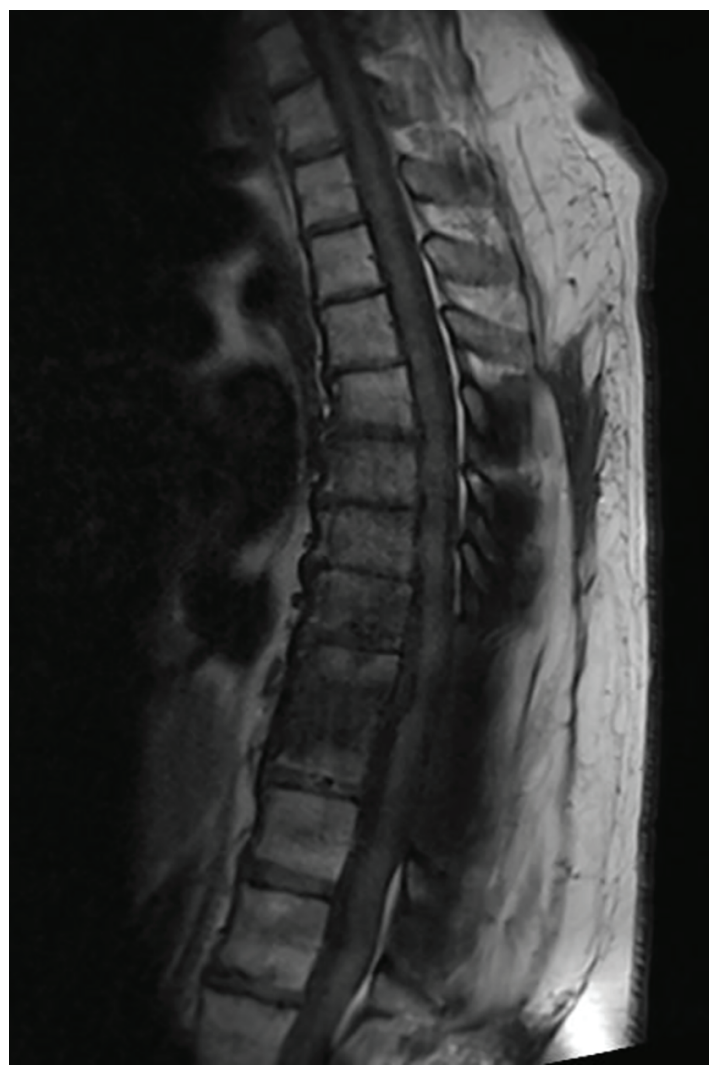

Figure 4. Infection and fusion at T9-T10.

discussing this case with all concerned departments in our institute (KKH, NGHA, Jeddah); the first scenario suggests that the infection was due to contamination from the epidural injection procedure which rapidly progressed to an acute case of spondylodiscitis. The second scenario suggests that an infection was already present and subclinical before the injection of the analgesic, and that the steroid injected in the epidural space as a pain control measure reduced the immunity at that area and gave a chance for the already existent infection to flourish. The third scenario is that the peptococcus being a mouth commensal got access to the blood of the patient simultaneously as the injection was given and found the condition favorable at the demonstrated infection site due to steroids although the patient did not give any history of endodontic procedure at or shortly before the epidural injection.

Peptococcus is a gram positive coccus anerobe that is found as normal mouth commensal in the mouth of human beings. It is not pathological when in natural sites. It could be involved in tissue infection such as fasciitis and also in bone infection as demonstrated in our reported case.

The incidence of pyogenic spondylodiscitis is somewhere around 1:250,000 to $1: 100,000$ per annum with a mortality rate of $2-17 \%$ [1]. Pyogenic spondylodiscitis represents $2-7 \%$ of all cases of osteomyelitis [2]. The presenting symptoms are usually lower back pain with or without constitutional symptoms [3]. Lower back pain, being the most common presentation in spine clinics, may be regarded with a low suspicion index especially there are no other symptoms observed that could raise the clinician's suspicion. All of the above mentioned factors could delay the diagnosis of this condition [4].

Once the diagnosis of spondylodiscitis is suspected attempts to isolate the causing organism should be started. Multiple blood cultures are recommended before more invasive measures are taken to isolate the causative agent [5]. In cases where blood cultures are negative then aspiration/biopsy under radiology guidance is indicated. This might not always close the case. In fact, some studies demonstrated that positive cultures from biopsy to diagnose spondylodiscitis may be as low as $32 \%[6]$.

The most frequently isolated organism in pyogenic spondylodiscitis is Staphylococcus aureus [7] and to a lesser extent streptococcus $\beta$ hemolytic species. Anerobic bacteria causing spondylodiscitis are very rare [8].

MRI has a sensitivity and specificity of $96 \%$ and $94 \%$, respectively, for diagnosing pyogenic spondylodiscitis $[3,6]$. MRI should always be sought when spondylodiscitis is suspected.

Antibiotics are to be empirically started immediately upon suspension of spondylodiscitis. They should cover staphylococcus and methicillin-resistant strains [2]. After cultures and sensitivity reports are obtained, antibiotics should be changed accordingly. The optimum period of time in which to administer parenteral antibiotics is not well defined. Higher rates of failure of treatment were reported with parenteral antibiotics administration less than 4 weeks [2].

Surgical treatment might be indicated in cases of pyogenic spondylodiscitis [1]. The surgical approach usually involves debridement, sampling of the affected area for microbiological and antibiotic sensitivity study, possible decompression and possible fixation. The approach could be anterior or posterior. In our reported case, the surgical approach was posterior with fixation.

Paraplegia is reported with a prevalence of $5 \%$ in some studies [7]. In this case, the patient recovered completely in contrast to our reported case that is still paraplegic 4 months after she was diagnosed.

\section{Conclusion}

Spondylodiscitis is a poorly understood condition which may be devastating if not discovered and treated optimally. A high index of suspicion should always be present. With the advancement of invasive interventions and their increasing indications and uses, such as epidural injection, catheters, punctures, etc., strict aseptic measures should be followed. Prophylactic antibiotic administration in endodontic procedures should always be administered.

\section{References}

1. Zarghooni K, Rollinghoff M, Sobottke R, Eysel P. Treatment of spondylodiscitis. Int Orthop. 2012;36(2):405411.

2. Pola E, Logroscino CA, Gentiempo M, Colangelo D, Mazzotta V, Di Meco E, Fantoni M. Medical and surgi- 
cal treatment of pyogenic spondylodiscitis. Eur Rev Med Pharmacol Sci. 2012;16(Suppl 2):35-49.

3. Lee SJ, Choi EJ, Nahm FS. Spondylodiscitis after Cervical Nucleoplasty without Any Abnormal Laboratory Findings. Korean J Pain. 2013;26(2):181-185.

4. Gurbuz MS, Berkman MZ. Spondylodiscitis occurring after diagnostic lumbar puncture: a case report. Case Rep Infect Dis. 2013;2013:843592.

5. Aagaard T, Roed C, Dragsted C, Skinhoj P. Microbiological and therapeutic challenges in infectious spondylodiscitis: a cohort study of 100 cases, 2006-2011. Scand J Infect Dis. 2013;45(6):417-424.
6. Sehn JK, Gilula LA. Percutaneous needle biopsy in diagnosis and identification of causative organisms in cases of suspected vertebral osteomyelitis. Eur J Radiol. 2012;81(5):940-946.

7. Valancius K, Hansen ES, Hoy K, Helmig P, Niedermann $\mathrm{B}$, Bunger $\mathrm{C}$. Failure modes in conservative and surgical management of infectious spondylodiscitis. Eur Spine J. 2013;22(8):1837-1844.

8. Saeed MU, Mariani P, Martin C, Smego RA, Jr., Potti A, Tight R, Thiege D. Anaerobic spondylodiscitis: case series and systematic review. South Med J. 2005;98(2):144148. 